\title{
Ist die Zukunft des Spitals ambulant?
}

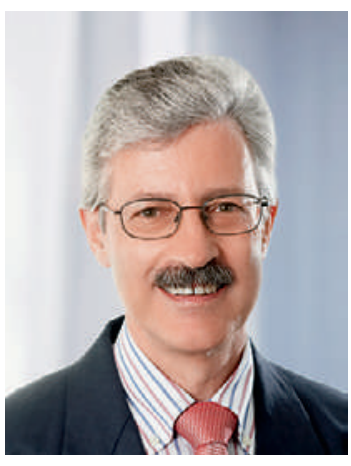

Früher wurden Kranke über einen längeren, teilweise sehr langen, Zeitraum im Spital versorgt, da es keine alternativen Unterbringungsmöglichkeiten gab. Dies war die Zeit ausgedehnter Aufenthalte in Sanatorien sowie umfangreicher Untersuchungen. Anhand der Ergebnisse bestimmten die Ärzteteams die Untersuchung für den nächsten Tag. Im Zuge der technischen Entwicklung, aber auch auf Wunsch der Patientinnen und Patienten, schnell wieder zu Hause zu sein sowie angesichts der Verknappung der finanziellen Mittel hat sich die Spitaltätigkeit in den vergangenen zwanzig Jahren stark verändert. Dies betrifft sowohl die spezialisierten und Notfallkonsultationen als auch die Durchführung komplexer diagnostischer und therapeutischer Verfahren.

\section{Mittelfristig werden wir nicht darum herum- kommen, uns intensiv mit der spitalambulanten Finanzierung auseinanderzusetzen.}

Um ambulante Leistungen von hoher Qualität anbieten zu können, muss ein Spital heute zahlreiche Anforderungen erfüllen. Neben der medizinisch-pflegerischen Kompetenz ist eine optimale Organisation der verschiedenen Abläufe notwendig. Alle vorgelagerten Massnahmen einer spitalambulanten Behandlung müssen in der richtigen und zeitlich bulanten Behandlung. Diese Einstellung hat sich geändert: Die allgemeine Beschleunigung des Lebens sowie die $\mathrm{Zu}$ nahme des Leistungsdrucks am Arbeitsplatz führten dazu, dass Patienten bei einem gesundheitlichen Problem oft eine schnelle Genesung wünschen, um ihre Berufstätigkeit möglichst bald fortführen zu können.

Die Finanzierung der ärztlichen Leistungen in der Schweiz setzt sich aus einem dualen Tarifsystem im Spitalbereich und einem monistischen im Praxisbereich zusammen. Die Tarifstrukturen SwissDRG und TARMED folgen einer unterschiedlichen Logik. Diese Tatsache setzt teilweise Anreize, bestimmte medizinische Prozeduren ambulant oder stationär durchzuführen. In dieser Hinsicht scheint die Reform der Spitalfinanzierung des Jahres 2012 keine bemerkbare Veränderung der Verhaltensweisen herbeigeführt zu haben, da sich die Tendenz zur Ausweitung der ambulanten Leistungen fortsetzt (siehe Artikel Seite 1443 in dieser Ausgabe). Mittelfristig werden wir nicht darum herumkommen, uns intensiv mit der Finanzierung der ambulanten Tätigkeit von Spitälern auseinanderzusetzen. Eine mögliche Lösung könnte eine einheitliche Pauschale für bestimmte Behandlungen sein, unabhängig davon, ob diese stationär oder ambulant erbracht wurden.

Der behandelnde Arzt ist und bleibt am besten in der Lage, die passende Art der Durchführung einer diagnostischen oder therapeutischen Massnahme dem Patienten vorzuschlagen. Auch wenn es nützlich ist, bestimmte Machbarkeitskriterien für die ambulante Durchführung eines Eingriffs aufzustellen, bleibt die menschliche Beziehung stets im Mittelpunkt einer solchen Entscheidung, denn jede Patientin und jeder Patient hat seine ganz persönliche Situation.

Ob sie stationär oder ambulant behandelt werden - die Patienten erwarten von uns Professionalität, Kompetenz und Organisationsvermögen. Sie wünschen sich ausreichend Raum für den persönlichen Austausch, wollen ernst genom-

\section{Ob stationär oder ambulant - die Patienten erwarten Professionalität, Kompetenz und Organisationsvermögen.}

abgestimmten Reihenfolge durchgeführt werden. Einerseits, um eine Gesamteinschätzung der Risiken aus medizinischer Sicht zu ermöglichen, andererseits, um die Patienten ausführlich zum empfohlenen Verfahren zu informieren und ihnen genügend Zeit für die Entscheidung zu lassen. Aber genauso wichtig ist die Nachbetreuung: Haben die Patienten nach der Rückkehr nach Hause Schmerzen oder Fragen, müssen sie sich jederzeit mit ihrem Anliegen an eine kompetente Person wenden können.

Lange Zeit bevorzugten die Patienten, mit Ausnahme einzelner Unternehmer und Führungskräfte, die Annehmlichkeiten eines kurzen Spitalaufenthalts anstelle einer spitalam- men werden und ihr Einverständnis zu den vorgeschlagenen diagnostischen oder therapeutischen Massnahmen geben können. Stationäre Spitaleinrichtungen wird es immer brauchen, gleichzeitig werden aber Aufenthalte, die nicht zwingend stationär erbracht werden müssen, rationalisiert und die ambulanten Möglichkeiten weiterentwickelt. Vor diesem Hintergrund bieten Spitaleinrichtungen der sekundären Versorgung und mittlerer Grösse gewisse Vorteile.

Dr. med. Pierre-François Cuénoud, Vizepräsident der FMH, Departementsverantwortlicher Tarife und Gesundheitsökonomie Spitalärzte 